\title{
An isolated outbreak of diphtheria in South Africa, 2015
}

\author{
S. MAHOMED ${ }^{1 *}$, M. ARCHARY ${ }^{2}$, P. MUTEVEDZI ${ }^{2,3}$, Y. MAHABEER $^{1,4}$, \\ P. GOVENDER ${ }^{2}$, G. NTSHOE ${ }^{4}$, W. KUHN ${ }^{2}$, J. THOMAS ${ }^{3}$, A. OLOWOLAGBA $^{5}$,

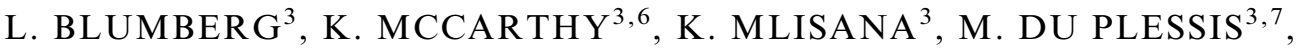 \\ A. VON GOTTBERG ${ }^{3,7}$ AND P. MOODLEY ${ }^{1,2}$ \\ ${ }^{1}$ School of Laboratory Medicine and Medical Sciences, College of Health Sciences, University of \\ KwaZulu-Natal, Durban, South Africa \\ ${ }^{2}$ KwaZulu-Natal Department of Health, Pietermaritzburg, South Africa \\ ${ }^{3}$ National Institute for Communicable Diseases, National Health Laboratory Service, Johannesburg, \\ South Africa \\ ${ }^{4}$ National Health Laboratory Service, Durban, South Africa \\ ${ }^{5}$ eThekwini Metropolitan Municipality Health Unit, Durban, South Africa \\ ${ }^{6}$ School of Public Health, University of the Witwatersrand, Johannesburg, South Africa \\ ${ }^{7}$ School of Pathology, University of the Witwatersrand, Johannesburg, South Africa
}

Received 18 January 2017; Final revision 10 March 2017; Accepted 29 March 2017;

first published online 8 May 2017

\section{SUMMARY}

An outbreak of respiratory diphtheria occurred in two health districts in the province of KwaZulu-Natal in South Africa in 2015. A multidisciplinary outbreak response team was involved in the investigation and management of the outbreak. Fifteen cases of diphtheria were identified, with ages ranging from 4 to 41 years. Of the 12 cases that were under the age of 18 years, $9(75 \%)$ were not fully immunized for diphtheria. The case fatality was $27 \%$. Ninety-three household contacts, 981 school or work contacts and 595 healthcare worker contacts were identified and given prophylaxis against Corynebacterium diphtheriae infection. A targeted vaccination campaign for children aged 6-15 years was carried out at schools in the two districts. The outbreak highlighted the need to improve diphtheria vaccination coverage in the province and to investigate the feasibility of offering diphtheria vaccines to healthcare workers.

Key words: Diphtheria antitoxin, epidemiology, vaccine-preventable diseases, surveillance.

\section{INTRODUCTION}

Respiratory diphtheria, usually caused by toxigenic strains of Corynebacterium diphtheriae, is a vaccinepreventable disease that can affect persons of all ages. Although the global incidence of diphtheria

\footnotetext{
* Author for correspondence: S. Mahomed, School of Laboratory Medicine and Medical Sciences, College of Health Sciences, University of KwaZulu-Natal, Postnet Suite 247, Private Bag X10, Musgrave Rd, Durban, 4001, South Africa. (Email: mahomeds@ukzn.ac.za)
}

has decreased significantly in the last three decades, the disease remains a problem, especially amongst young children in countries with low immunization coverage [1].

In South Africa, diphtheria-containing vaccines are part of the Expanded Programme on Immunization (EPI) for children and are available at no cost in the public health sector. A primary series of three vaccinations with diphtheria toxoid are given at 6,10 , and 14 weeks of age administered as a combination vaccine. At 18 months, a booster dose is given using the 
same vaccine as for the primary series. Since 2008, two additional booster doses with a reduced concentration of toxoid are recommended at 6 and 12 years of age [2].

KwaZulu-Natal is one of nine provinces in South Africa and is divided into 11 health districts. In 2014, according to the data from the District Health Information System which is used to manage routinely collected health service-based information [3], the coverage of the primary series diphtheria vaccinations in the province was $96 \%$, and the 18 month booster vaccination was administered to $83 \%$ of children at that age. The coverage for the 6 year and 12 year booster vaccinations was $54 \%$ and $20 \%$, respectively.

Since 2000, only seven cases of diphtheria have been reported in South Africa, the last of which was documented in 2010 [4]. In KwaZulu-Natal, the last reported diphtheria case was in 1989 [5].

Between March and June 2015, 15 cases of diphtheria were identified in two health districts in the KwaZulu-Natal Province in South Africa. We describe the clinical, laboratory, and public health aspects of this outbreak.

\section{METHODS}

\section{Case definitions}

After the first case was diagnosed, case definitions were drawn up as part of management guidelines to facilitate case finding. Cases were classified as suspect, probable, or confirmed (Table 1).

\section{Laboratory diagnosis}

Throat swabs from suspected cases were plated on 5\% horse blood agar and Hoyle's media (Diagnostic Media Products, National Health Laboratory Service, Johannesburg, South Africa) with an additional chocolate agar plate for tissue specimens. Nasal swabs from contacts and nasopharyngeal swabs collected during the enhanced surveillance were plated onto Hoyle's agar only. Plates were incubated for $48 \mathrm{~h}$. All Gram-positive bacilli that grew as black pigmented colonies on Hoyle's plates were identified using biochemical tests and MatrixAssisted Laser Desorption Ionization Time-of-Flight (MALDI-TOF) (bioMérieux S.A.). Toxin production and the presence/absence of the A and B subunits of the $C$. diphtheriae toxin (tox) gene were confirmed by the Elek test and real-time PCR, respectively
$[6,7]$. Multilocus sequence typing (MLST) was used to characterise the isolates [8]. The sequence types (STs) were extracted from whole genome data and compared with all available STs $(n=437)$ listed in the global MLST database (http://pubmlst.org/ cdiphtheriae/).

\section{Co-ordinated outbreak response}

A multidisciplinary provincial outbreak response team was established following identification and confirmation of the first case of diphtheria. The team included senior district and municipal health management, communicable disease control (CDC) managers and co-ordinators, clinicians, microbiologists, an epidemiologist, and pharmacy managers. In addition the following departments were represented: school health and EPI, occupational health, health promotion, environmental health, infection prevention and control, and public health medicine. The team met weekly, discussed cases, presented findings and activities of field teams and agreed upon response measures. The provincial CDC unit was responsible for the co-ordination of outbreak response activities, and the district outbreak response teams were responsible for the implementation of these activities.

The National Institute for Communicable Diseases (NICD) Outbreak Response Unit provided support to through the compilation of case definitions and management guidelines, collation of epidemiological data on each case, and provision of a daily situational report. The NICD operated a 24-h 'hotline' to assist with case identification, management, and reporting.

\section{Case detection}

The district outbreak response teams alerted staff at public and private healthcare facilities to be aware of the possibility of diphtheria. Media reports facilitated increased public awareness. Community caregivers (non-professional individuals providing care within communities) were trained on the signs and symptoms of diphtheria.

\section{Clinical management and case investigation}

A clinical protocol on the diagnostic criteria and acute management of suspected diphtheria cases was distributed to all healthcare facilities in the province. Each suspected case was discussed with a provincial paediatric infectious disease specialist (M.A.) who 
Table 1. Case definitions implemented during the diphtheria outbreak in KwaZulu-Natal, 2015

\begin{tabular}{|c|c|}
\hline $\begin{array}{l}\text { Classification } \\
\text { of cases }\end{array}$ & Definition \\
\hline Suspect & $\begin{array}{l}\text { Any person presenting with: pharyngitis, nasopharyngitis, tonsillitis, laryngitis, tracheitis (or any } \\
\text { combination of these), where fever is absent or low grade, AND one or more of the following: } \\
\text { - Adherent pseudo-membrane which bleeds if manipulated or dislodged } \\
\text { - Features suggestive of severe diphtheria, including: stridor, bull-neck, cardiac complications } \\
\text { (myocarditis, acute cardiac failure and circulatory collapse), acute renal failure }\end{array}$ \\
\hline Probable & $\begin{array}{l}\text { A person who meets the suspect case definition for respiratory diphtheria plus one of the following: } \\
\text { - Isolation of } C \text {. diphtheriae/C. ulcerans/C. pseudotuberculosis but toxigenicity status has not yet been } \\
\text { confirmed } \\
\text { - Has an epidemiologic link with a laboratory-confirmed case or carrier } \\
\text { OR } \\
\text { A person presenting with mild respiratory symptoms with no membrane or other presentations of } \\
\text { diphtheria, but has an epidemiologic link to a laboratory-confirmed case or carrier }\end{array}$ \\
\hline Confirmed & $\begin{array}{l}\text { A person who meets the suspect case definition for respiratory diphtheria and is laboratory confirmed } \\
\text { OR } \\
\text { A person presenting with mild respiratory symptoms with no membrane or other presentations of } \\
\text { diphtheria and is laboratory confirmed }\end{array}$ \\
\hline $\begin{array}{l}\text { Asymptomatic } \\
\text { carrier }\end{array}$ & A person with no symptoms but has laboratory confirmation of a toxigenic strain \\
\hline Discarded & $\begin{array}{l}\text { A suspected or probable case in whom other compatible organisms are isolated or if } C \text {. diphtheriae/C. } \\
\text { ulcerans/ C. pseudotuberculosis is isolated but is confirmed to be a non-toxigenic strain }\end{array}$ \\
\hline
\end{tabular}

evaluated the patient's clinical presentation, and assessed the need to administer anti-toxin. Suspected cases were managed at the hospital where they presented. Suspected cases were treated for 14 days with either intravenous soluble penicillin $(50000 \mathrm{IU} / \mathrm{kg})$, or azithromycin $(10 \mathrm{mg} / \mathrm{kg})$. Patients who required ventilation were transferred to a tertiary-level hospital with intensive care facilities. When a suspected case was identified, a case investigation form was completed with demographic and medical history, clinical management and outcome. Vaccination status was ascertained by review of Road-to-Health card. If this card was not available, vaccination status was reported as 'unknown'.

Diphtheria antitoxin (DAT) was first purchased from India, and subsequently donated by the Republic of Japan (Vaccine Business Section, Sales Division, Kaketsuken, Chemo-Sero-Therapeutic Research Institute), but this was not available when the first three cases were identified.

\section{Contact tracing}

Following identification of a probable case, and before laboratory confirmation, contact tracing was initiated by a local area-based outbreak response team led by CDC co-ordinators. A contact was defined as someone who had direct physical contact with a case, or who either lived in the same household as the case or spent a significant amount of time with the case, for example learners sharing the same classroom or work colleagues sharing the same office. The CDC co-ordinator, together with the infection control practitioner at the healthcare facility identified any healthcare worker that was involved in the management of cases, including paramedical staff who transported cases between healthcare facilities. Nasal swabs were obtained from contacts. A 'contact investigation form' was completed for all contacts to obtain demographic details, and their clinical history and details of all possible contacts.

\section{Enhanced surveillance}

Enhanced surveillance was done in five primary healthcare clinics, in the areas of the eThekwini Health District from which the majority of cases emanated. This surveillance commenced in June 2015 and aimed to identify cases of diphtheria of mild-tomoderate severity over a 6-week period. Trained nurses obtained nasopharyngeal swabs from adults and children with symptoms of an upper respiratory tract infection (cough, fever, runny nose). 


\section{Targeted vaccination}

A targeted vaccination campaign was commenced at schools closest to the areas from which the cases emanated. Learners aged 6-15 years were targeted to receive a booster dose of diphtheria vaccine (given in combination with tetanus as Diftavax ${ }^{\circledR}$ ). Learners were provided with consent forms, and those learners who obtained signed parental consent were vaccinated.

\section{Ethics}

Permission was obtained from the KwaZulu-Natal Department of Health Research and Ethics Committee to publish patient data. The NICD has ethics approval from the Human Research Ethics Committee of the University of the Witwatersrand to publish data from outbreak investigations (M160667).

\section{RESULTS}

\section{Index case}

On 15 March 2015, an 8-year-old male presented to a regional hospital in eThekwini Health District in respiratory distress with neck swelling, drooling, and difficulty swallowing. On examination, he had massively enlarged tonsils with a pseudo-membrane over the tonsils and soft palate. He was transferred to a tertiary hospital where he underwent a tracheostomy. The otorhinolaryngologist (W.K.) who attended to the patient made a clinical diagnosis of diphtheria. Endotracheal swabs taken yielded $C$. diphtheriae. The patient spent 4 days in intensive care and was transferred to the referring hospital following clinical improvement. The confirmation of the diagnosis in this patient led to the activation of the district, provincial and national outbreak response structures as described above. At the time, DAT was not available in the country.

\section{Descriptive epidemiology}

After the first case, there were three more cases in eThekwini Health District over the ensuing 2 weeks. Four weeks later, the first case was reported from Ugu Health District which is approximately $120 \mathrm{~km}$ away from eThekwini Health District (Fig. 1). The last case was reported on 12 June 2015. In total there were 15 cases over a 14-week period (Fig. 2).
Twelve cases were from eThekwini Health District and three cases from Ugu Health District. There was no travel history amongst the cases or contacts interviewed.

The age of the patients ranged from 4 to 41 years with a median age of 10 . The majority $(n=7,47 \%)$ were in the age group 6-11 years. Nine of the cases $(60 \%)$ were male. Of those who were $<18$-years old $(n=12)$, the majority $(n=9,75 \%)$ were incompletely or never immunized. Two of the cases did not have immunization records, and were classified as unknown (Table 2).

The overall CFR (case-fatality ratio) was $27 \%(n=$ 4), with all deaths occurring in eThekwini Health District.

\section{Clinical presentation and management}

The mean time from onset of symptoms to admission was 3 days (range 1-5 days). The commonest clinical sign on presentation was tonsillitis/pharyngitis $(n=14$, $93 \%)$ followed by fever $(n=11,73 \%)$. Visualisation of a pseudo-membrane and the presence of a bull-neck was reported in 10 cases (67\%). Twelve of the 15 cases $(80 \%)$ presented with severe respiratory distress requiring acute airway management or monitoring. The two cases who presented with mild respiratory symptoms were fully immunized. Thirteen of the cases were HIV uninfected (Table 3).

Two patients required surgical airway management. Four additional cases required ventilation.

Myocarditis was diagnosed in four patients (27\%). This diagnosis was based on clinical, electrocardiogram and echocardiographic features. Myocarditis developed on average 14 days after admission to hospital and all 14 patients recovered without sequelae following medical management. Two of the four patients received DAT; 12 days and 4 days postadmission, respectively. An additional patient had a sudden unexpected death during the convalescence phase of the infection which was suspected to be secondary to an undiagnosed myocarditis. No postmortem was done to confirm this.

Three patients developed renal dysfunction. Two of these patients recovered with simple fluid therapy; one of whom had received DAT. One patient died from multi-organ failure with renal failure playing a major role.

Two patients developed a peripheral neuropathy, both patients presenting with a pseudo-bulbar palsy 


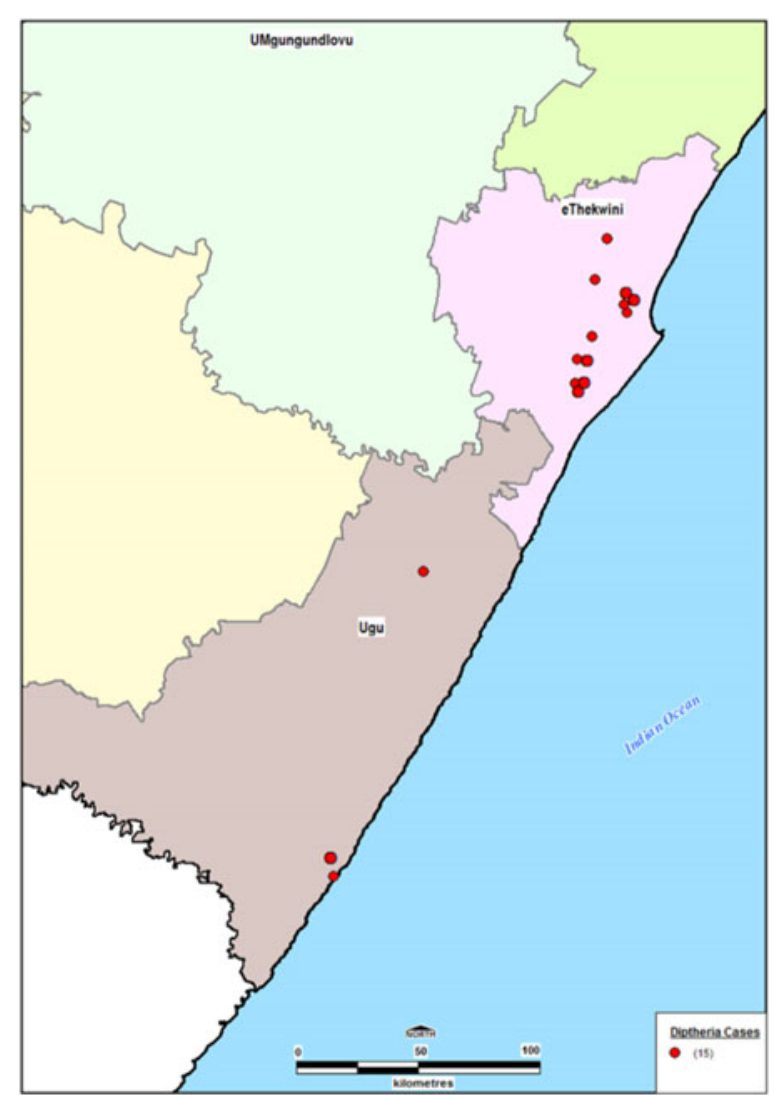

Fig. 1. Geographic location of 15 diphtheria cases identified during an outbreak in KwaZulu-Natal, 2015.

4 weeks after the initial admission. Neither patient received DAT.

A further two deaths occurred; one was attributed to systemic-related toxicity and the other demised at home before presentation to a healthcare facility.

\section{Laboratory results}

Specimens for culture were received from 13 of the 15 patients. The two cases without laboratory specimens included the case that demised at home and a case that was managed in a private hospital. Toxin production was confirmed in all isolates that carried the tox gene. Eleven of the 13 isolates were toxin positive and were classified as confirmed cases. Two of the cases with toxin-negative isolates were treated as probable cases as they were linked to asymptomatic carriers. One case-patient yielded two isolates: a toxin-positive isolate and a toxin-negative isolate.

An additional six toxin-positive isolates were from asymptomatic carriers epidemiologically linked to cases. This was carefully reviewed to exclude laboratory error. Two additional toxin-negative
C. diphtheriae isolates were found: one was a contact of a case, and one was from a patient with possible cutaneous diphtheria.

Two novel STs were identified among the outbreak isolates, none of which were related to any other STs listed in the global PubMLST database at the time of this analysis (http://pubmlst.org/cdiphtheriae/). All 17 toxin-positive isolates collected from cases and contacts during the outbreak were ST-378. The toxinnegative isolates were ST-395.

\section{Outbreak response}

\section{Contact tracing}

Ninety-three household contacts, 981 school or work contacts and 595 healthcare workers were identified. All of the healthcare workers received prophylactic antibiotics (azithromycin or erythromycin, depending on availability) and the diphtheria booster vaccine. All household contacts received prophylaxis and 88 $(95 \%)$ received the diphtheria booster vaccine. Two contacts (siblings of cases 5 and 13) progressed to developing diphtheria symptoms after receiving prophylaxis and were classified and managed as cases. There were no other cases emanating from the households, work places or schools at which contacts received prophylaxis.

\section{Targeted vaccination}

There are 805 schools in the eThekwini Health District with a population of 411977 in the targeted age group. Only $446(55 \%)$ of these schools were visited during the outbreak response resulting in a $52 \%$ immunization coverage. In Ugu Health District, there were 209 schools with a target population of 77406 . One hundred and eighty $(86 \%)$ schools were visited and $39 \%$ of the target population was vaccinated. The targeted vaccination campaign occurred in May and June, during epidemiological weeks $21-$ 25 . During the campaign, the four cases (cases 1114) that were identified were not from the schools, or communities near the schools, that had been visited as part of the campaign. There were no further cases reported after the targeted vaccination campaign ended.

\section{Community participation and mobilization}

A total of 195 community care givers were trained on the signs and symptoms of diphtheria in the two affected districts. 


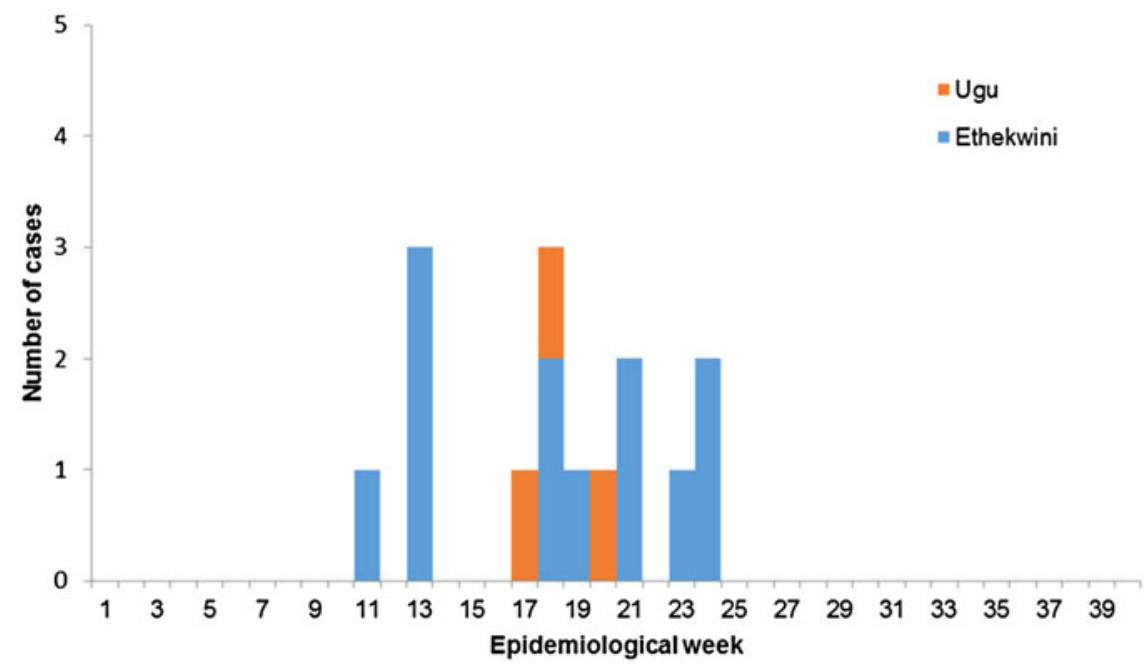

Fig. 2. Number of diphtheria cases by week of illness and district, KwaZulu-Natal, 2015.

Table 2. Demographic characteristics and laboratory results of 15 diphtheria cases identified during an outbreak in KwaZulu-Natal, 2015

\begin{tabular}{|c|c|c|c|c|c|c|c|c|}
\hline Case & $\begin{array}{l}\text { Case } \\
\text { classification }\end{array}$ & Gender & $\begin{array}{l}\text { Age at } \\
\text { diagnosis }\end{array}$ & District & $\begin{array}{l}\text { Vaccination } \\
\text { status }\end{array}$ & $\begin{array}{l}\text { Throat swab } \\
\text { culture result }\end{array}$ & $\begin{array}{l}\text { Elek test } \\
\text { result }\end{array}$ & $\begin{array}{l}\text { Epidemiological } \\
\text { considerations }\end{array}$ \\
\hline 1 & Confirmed & Male & 8 & eThekwini & Incomplete & Positive & Positive & None \\
\hline 2 & Confirmed & Female & 8 & eThekwini & Incomplete & Positive & Positive & Possible link to case 1 \\
\hline 3 & Confirmed & Male & 9 & eThekwini & Incomplete & Positive & Positive & None \\
\hline 4 & Suspect & Male & 11 & eThekwini & Incomplete & Not done & N/A & None \\
\hline 5 & Confirmed & Female & 9 & Ugu & Unknown & Positive & Positive & Linked to case 6 \\
\hline 6 & Confirmed & Female & 5 & Ugu & Unknown & Positive & Positive & Sibling of case 5 \\
\hline 7 & Confirmed & Female & 41 & eThekwini & Not available & Positive & Positive & None \\
\hline 8 & Confirmed & Male & 13 & eThekwini & Incomplete & Positive & Positive & None \\
\hline 9 & Confirmed & Male & 17 & eThekwini & Incomplete & Positive & Positive & None \\
\hline 10 & Probable & Male & 11 & $\mathrm{Ugu}$ & Up to date for age & Positive & Negative & $\begin{array}{l}\text { Linked to } \\
\text { asymptomatic carriers }\end{array}$ \\
\hline 11 & Probable & Male & 8 & eThekwini & Incomplete & Not done & N/A & $\begin{array}{l}\text { Linked to an } \\
\text { asymptomatic carrier }\end{array}$ \\
\hline 12 & Confirmed & Male & 21 & eThekwini & Unknown & Positive & Positive & None \\
\hline 13 & Confirmed & Female & 13 & eThekwini & Incomplete & Positive & Positive & Linked to case 14 \\
\hline 14 & Confirmed & Male & 4 & eThekwini & Up to date for age & Positive & Positive & Sibling of case 13 \\
\hline 15 & Suspect & Female & 35 & eThekwini & Incomplete & Negative & N/A & None \\
\hline
\end{tabular}

\section{Surveillance}

Nasopharyngeal swabs were obtained from a total of 782 adults and children with symptoms of an upper respiratory tract infection. None of these specimens tested positive for $C$. diphtheriae.

\section{DISCUSSION}

The majority $(75 \%)$ of the cases in this outbreak of diphtheria were incompletely immunized and the outbreak occurred in a population with low immunization coverage of the 6- and 12-year booster vaccinations. The predominant populations affected in diphtheria outbreaks are children and adults with waning immunity [9]. In recent diphtheria outbreaks in Nigeria and Brazil, the majority of the cases were partially immunized or unimmunized - 98\% and $62 \%$, respectively $[10,11]$. KwaZulu-Natal has a high burden of childhood mortality, mainly from diarrhea and pneumonia [12]; and diseases that do not contribute to this high morbidity and mortality are 
Table 3. Clinical features and management of 15 diphtheria cases identified during an outbreak in KwaZulu-Natal, 2015

\begin{tabular}{|c|c|c|c|c|c|c|c|c|c|c|c|c|}
\hline \multirow[b]{2}{*}{ Case } & \multirow{2}{*}{$\begin{array}{l}\text { Duration of } \\
\text { symptoms prior to } \\
\text { admission (days) }\end{array}$} & \multirow[b]{2}{*}{$\begin{array}{l}\text { HIV } \\
\text { status }\end{array}$} & \multicolumn{6}{|c|}{ Clinical features } & \multicolumn{2}{|c|}{ Toxin-mediated complications } & \multirow[b]{2}{*}{$\begin{array}{l}\text { Airway } \\
\text { support }\end{array}$} & \multirow[b]{2}{*}{$\begin{array}{l}\text { DAT } \\
\text { administered }\end{array}$} \\
\hline & & & Fever & $\begin{array}{l}\text { Tonsillitis/ } \\
\text { pharyngitis }\end{array}$ & $\begin{array}{l}\text { Pseudo- } \\
\text { membrane }\end{array}$ & $\begin{array}{l}\text { Neck } \\
\text { swelling }\end{array}$ & $\begin{array}{l}\text { Respiratory } \\
\text { distress }\end{array}$ & $\begin{array}{l}\text { Renal } \\
\text { dysfunction }\end{array}$ & Myocarditis & Neuropathy & & \\
\hline $1^{*}$ & 5 & Negative & & & & & & & Suspected & & $\mathrm{T}$ & \\
\hline 2 & 1 & Negative & & & & & & & & & & \\
\hline 3 & 3 & Negative & & & & & & & & & & \\
\hline 4 & 5 & Negative & & & & & & & & & & \\
\hline 5 & 4 & Negative & & & & & & & & & $\mathrm{T}$ & \\
\hline 6 & 1 & Negative & & & & & & & & & I & $\dagger^{\dagger}$ \\
\hline 7* & 2 & Unknown & & & & & & & & & I & \\
\hline $8^{*}$ & 3 & Negative & & & & & & & & & I & \\
\hline 9 & 4 & Negative & & & & & & & & & $\mathrm{T}$ & \\
\hline 10 & 1 & Negative & & & & & M & & & & & \\
\hline $12^{*}$ & N/A & Unknown & & & & & & & & & $\mathrm{T}$ & \\
\hline 13 & 4 & Negative & & & & & & & & & & \\
\hline 14 & 1 & Negative & & & & & M & & & & & \\
\hline \multirow[t]{2}{*}{15} & 3 & Negative & & & & & & & & & $\mathrm{T}$ & \\
\hline & & & $11(73 \%)$ & $14(93 \%)$ & $10(67 \%)$ & $10(67 \%)$ & $12(80 \%)$ & $3(20 \%)$ & $4(30 \%)$ & $2(13 \%)$ & $\begin{array}{l}\text { T:5 }(33 \%) \\
\text { I: } 3(20 \%)\end{array}$ & $6(40 \%)$ \\
\hline
\end{tabular}

M, Mild disease; I, intubated; T, tracheostomy. *Patient demised.

$\dagger$ Delayed administration of DAT. 
often neglected. Prior to this outbreak, the low coverage of the diphtheria booster vaccinations in all districts in the province had not been enhanced through catch-up campaigns. In the absence of programmatic booster vaccinations after childhood, waning immunity increases the susceptibility of older children as well as adults [13].

In countries with high vaccination coverage, it is postulated that diphtheria can occur as a result of the importation of new strains of toxigenic C. diphtheriae [9]. This assumes a variation in antigenic structure of the toxin for which vaccination does not offer protection. Although the novel ST (378) of the isolates makes importation of C. diphtheriae unlikely; it may have been imported from a country with poor microbiological diphtheria surveillance. However, the lack of travel history amongst cases and contacts decreases this likelihood. The outbreak was restricted to only two districts in the province which are approximately $120 \mathrm{~km}$ apart. It is probable that there was unreported travel history amongst the cases or controls that linked these two districts. It is unclear why there were no cases from the surrounding districts especially since eThekwini Health District is the most populous district in the province with a wide transport network. The outbreak occurred concurrently with the influenza season in KwaZuluNatal, and mild cases of diphtheria may have been misdiagnosed. However, the enhanced surveillance at clinics in eThekwini Health District yielded no positive $C$. diphtheriae isolates.

Crowding and poor hygiene can facilitate disease transmission [14]. Although many of the cases emanated from areas with poor socio-economic conditions, we did not observe a high transmission rate of the disease. The epidemic curve also highlights the sporadic nature of the outbreak. Cutaneous diphtheria is known to be a reservoir of circulating strains which may become toxigenic $[15,16]$. Nevertheless, this was not the case during the KZN outbreak as the non-toxigenic isolates belonged to an unrelated genotype.

Underlying immunosuppression did not appear to be a contributor to the transmission or manifestation of diphtheria amongst the cases. Although the outbreak occurred in an area with a high HIV prevalence, none of the patients were known to be HIV infected. The $27 \%$ case fatality in this outbreak is higher than other recent outbreaks reported in Nigeria $(21 \%)$ and India $(20 \%)[10,17]$. Only one of the cases demised prior to the availability of DAT. One of the deaths may have been linked to poor access to healthcare services.

Contact tracing was successfully conducted for all cases. Vaccination coverage of $100 \%$ was not achieved among contacts because in the initial phase of the outbreak, it was not clearly communicated that all contacts, irrespective of age, should receive a diphtheria booster vaccination. The high number of healthcare workers that were identified as contacts is disproportionate to the number of cases during the outbreak. In some areas, healthcare workers were concerned about the possibility of acquiring the disease and identified themselves as contacts even though they were not likely to have been in contact with a diphtheria case. Whilst it would have been ideal to offer all healthcare workers a booster dose of diphtheria vaccine, there was insufficient stock of the vaccine to do so. The nasal swabs taken during contact tracing were not ideal specimens as nasopharyngeal specimens are recommended for identifying asymptomatic carriers. The correct flexible swabs are not routinely available in our healthcare setting.

We were unable to extend the immunization to all learners that would have been eligible to receive a diphtheria booster vaccination (anyone who missed a booster dose) due to insufficient vaccine stock. Although a large proportion of schools were reached as part of the targeted vaccination drive, there were challenges encountered. First, the school health teams had difficulty negotiating time to vaccinate learners as the schools had commenced with mid-year examinations. Second, many learners did not bring back signed consent forms and in some instances learners older than 12 years did not assent to being vaccinated. Despite these limitations, the vaccination campaign is likely to have contributed to the halt in transmission of diphtheria in the affected districts.

\section{CONCLUSION}

Maintaining high coverage of diphtheria booster vaccines in adolescence is vital to prevent outbreaks of this disease. There is an urgent need to improve this immunization coverage in KwaZulu-Natal. School vaccination programmes should be ongoing until all districts in the province have achieved an immunization coverage of at least $80 \%$. At a national level, the feasibility of offering all teenagers and adults a diphtheria vaccine booster if they have not been immunized against diphtheria in the previous 10 years should be considered. Ten-yearly diphtheria 
booster vaccination should be included in the routine package of services offered to all healthcare workers. In areas with sub-optimal diphtheria vaccine coverage, a stock of DAT should be readily available to facilitate optimal management of diphtheria cases.

\section{ACKNOWLEDGEMENTS}

The authors would like to acknowledge the eThekwini and Ugu District CDC co-ordinators (Mr Mbuthu, Ms Ndlovu, Mr Mhlongo, Mr M Hadebe and Ms Mlambo) and their teams, Ms Gumede and Ms Fynn, Ms Dladla, Dr Z Banoo, the school health teams, Samuel Candice; and all other members of the provincial and district outbreak response teams for their role in managing the outbreak. They would also like to acknowledge laboratory support from Valentino Horne, Diagnostic Media Production Green Point, National Health Laboratory Service, Cape Town; staff at the Centre for Respiratory Diseases and Meningitis, NICD, Johannesburg; and the technical assistance from the World Health Organization.

\section{DECLARATION OF INTEREST}

None.

\section{REFERENCES}

1. World Health Organization. Weekly Epidemiological Record. Geneva: World Health Organization, 2006, pp. 24-32.

2. National Department of Health. Vaccinator's Manual "Immunisation That Works" Expanded Programme on Immunisation in South Africa (EPI-SA). Pretoria: South African National Department of Health, 2012.

3. National Department of Health. District Health Management Information System (DHMIS) Standard Operating Procedures: Facility Level. Pretoria: South African National Department of Health, Pretoria, 2012.

4. National Institute for Communicable Diseases. Communicable Diseases Communique February 2010.
Johannesburg: National Institute for Communicable Diseases, 2010.

5. Jeena PM, Wesley AG, Coovadia HM. Infectious diseases at the paediatric isolation units of Clairwood and King Edward VIII hospitals, Durban. South African Medical Journal 1998; 88: 867-872.

6. Efstratiou AMP. WHO Manual for the Laboratory Diagnosis of Diphtheria. Document ICP-EPI 038(C). Geneva, Switzerland: World Health Organization, 1994.

7. Mothershed EA, et al. Development of a real-time fluorescence PCR assay for rapid detection of the diphtheria toxin gene. Journal of Clinical Microbiology 2002; 40: 4713-4719.

8. Bolt F, et al. Multilocus sequence typing identifies evidence for recombination and two distinct lineages of Corynebacterium diphtheriae. Journal of Clinical Microbiology 2010; 48: 4177-4185.

9. Galazka A, Dittmann S. The changing epidemiology of diphtheria in the vaccine Era. Journal of Infectious Diseases 2000; 181(Suppl. 1): S2-S9.

10. Besa NC, et al. Diphtheria outbreak with high mortality in northeastern Nigeria. Epidemiology and Infection 2014; 142: 797-802.

11. Santos LS, et al. Diphtheria outbreak in Maranhão, Brazil: microbiological, clinical and epidemiological aspects. Epidemiology and Infection 2015; 143: 791-798.

12. Massyn NPN, Padarat A, Barron P, Day C, editors. District Health Barometer 2014/15. Durban: Health Systems Trust, 2015.

13. Mattos-Guaraldi AL, et al. Diphtheria remains a threat to health in the developing world: an overview. Memórias do Instituto Oswaldo Cruz 2003; 98: 987-993.

14. Quick ML, et al. Risk factors for diphtheria: a prospective case-control study in the Republic of Georgia, 1995-1996. Journal of Infectious Diseases 2000; 181: S121-S129.

15. Bowler I, et al. Diphtheria - the continuing hazard. Archives of Disease in Childhood 1988; 63: 194-210.

16. Hart PE, et al. Cutaneous and pharyngeal diphtheria imported from the Indian subcontinent. Postgraduate Medical Journal 1996; 72: 619-620.

17. Das PP, et al. Recent outbreaks of diphtheria in Dibrugarh district Assam, India. Journal of Clinical and Diagnostic Research Published online: July 2016; 10: DR01-DR03. 